\title{
Inverse Analysis of Crack in Fixed-Fixed Structure by Neural Network with the Aid of Modal Analysis
}

\author{
Dhirendranath Thatoi ${ }^{1}$ and Prabir Kumar Jena ${ }^{2}$ \\ ${ }^{1}$ Department of Mechanical Engineering, I.T.E.R, Bhubaneswar 751030, Odisha, India \\ ${ }^{2}$ Department of Mechanical Engineering, Silicon Institute of Technology, Bhubaneswar 751024, Odisha, India
}

Correspondence should be addressed to Prabir Kumar Jena; prabirkumarjena07@gmail.com

Received 6 December 2012; Accepted 11 February 2013

Academic Editor: Kyong Joo Oh

Copyright (C) 2013 D. Thatoi and P. K. Jena. This is an open access article distributed under the Creative Commons Attribution License, which permits unrestricted use, distribution, and reproduction in any medium, provided the original work is properly cited.

\begin{abstract}
In this research, dynamic response of a cracked shaft having transverse crack is analyzed using theoretical neural network and experimental analysis. Structural damage detection using frequency response functions (FRFs) as input data to the backpropagation neural network (BPNN) has been explored. For deriving the effect of crack depths and crack locations on FRF, theoretical expressions have been developed using strain energy release rate at the crack section of the shaft for the calculation of the local stiffnesses. Based on the flexibility, a new stiffness matrix is deduced that is subsequently used to calculate the natural frequencies and mode shapes of the cracked beam using the neural network method. The results of the numerical analysis and the neural network method are being validated with the result from the experimental method. The analysis results on a shaft show that the neural network can assess damage conditions with very good accuracy.
\end{abstract}

\section{Introduction}

Vibration-based methods for detection of cracks offer some advantages over conventional methods. This methodology can help to determine the location and size of the cracks from the vibration data collected from the cracked beam structure. Development of cracks in a vibrating structure leads to reduction in the stiffness and increase in its damping [1] which, in turn, gives rise to a change in natural frequencies and mode shapes. So it may be possible to estimate the location and size of the cracks by measuring changes in the vibration parameters.

Tada et al. [2] have proposed the basis for calculation of compliance matrix for different types of beams. Sekhar and Prabhu [3] have derived a method to calculate the vibration characteristics using a model based on finite element. Paviglianiti et al. [4] have devised a scheme for detecting and isolating sensor faults in industrial robot manipulators. They have adopted a procedure for decoupling of the disturbance effect from the effect of the fault generated in the system. The dynamics of the proposed scheme has been improved by using radial basis functions neural network. Behera et al. [5] have studied the vibration characteristics of a shaft with two open cracks rotating in a fluid medium by using the influence coefficient method to find frequency of the cracked shaft and frequency contours with respect to crack depths and locations. Wang et al. [6] have investigated the bending and torsional vibration of a fiber reinforced composite cantilever with a surface crack. They have suggested that the coupling of bending and torsion is the result of material properties or the surface crack. Lee [7] has presented a method to identify multiple cracks in a beam by modeling the cracks as rotational springs. He has claimed that the results are in excellent agreement with the actual ones. Artificial neural networks (ANN) can be used as an alternative effective tool for solving the inverse problems because of the pattern-matching capability. The results of ANN are quite encouraging and prove the robustness of the proposed damage assessment algorithm [8-10].

The paper is organized as following. In Section 2, a general framework to address local flexibility matrix at the vicinity of the crack is introduced. Neural controller mechanism for crack detection of the shaft with the aid of modal analysis is discussed in Section 3. Section 4 provides 


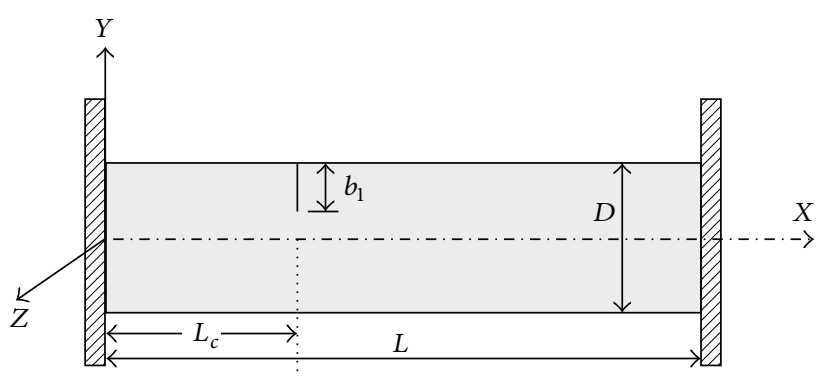

(a)

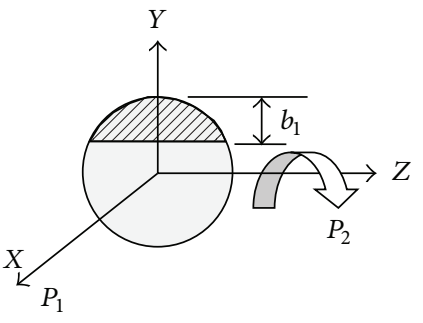

(b)

FIGURE 1: Geometry of the shaft. (a) Fixed-Fixed shaft, (b) cross-sectional view of the shaft.

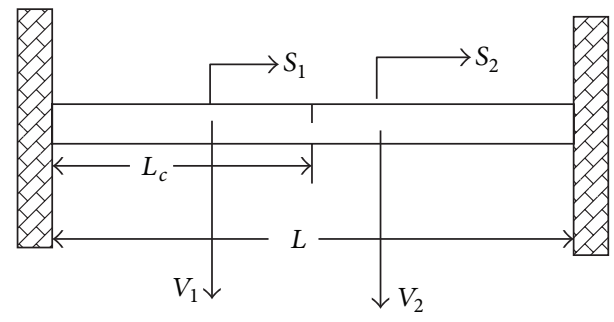

FIGURE 2: Shaft model.

a detailed description of the experimental method deployed, and the results obtained from this has been compared with that obtained from the theoretical and ANN results. A good agreement between the results has been observed.

\section{Problem Formulation and Theory}

2.1. Local Flexibility of a Cracked Beam under Bending and Axial Loading. The presence of a transverse surface crack of depth " $b_{1}$ " on a shaft of diameter $D$ introduces a local flexibility, which can be defined in matrix form, the dimension of which depends on the degrees of freedom. Here a $2 \times 2$ matrix is considered. A fixed-fixed shaft is subjected to axial force $\left(P_{1}\right)$ and bending moment $\left(P_{2}\right)$, shown in Figure 1, which is coupled to give both longitudinal and transverse motion. The cross-sectional view of the shaft is shown in Figure 1(b).

Using strain energy release rate, the local stiffness of a cracked shaft can be obtained in the matrix form as [2]

$$
K=\left[\begin{array}{ll}
K_{11} & K_{12} \\
K_{21} & K_{22}
\end{array}\right]=\left[\begin{array}{ll}
C_{11} & C_{12} \\
C_{21} & C_{22}
\end{array}\right]^{-1},
$$

where, $C_{i j}$ represents the compliance matrix.

\subsection{Analysis of Vibration Characteristics of the Cracked Shaft}

2.2.1. Free Vibration. A shaft of length " $L$ " with a crack of depth " $b_{1}$ " at a distance " $L_{c}$ " from the left-fixed end is considered (shown in Figure 1). Taking $S_{1}(x, t)$ and $S_{2}(x, t)$ as the amplitudes of longitudinal vibration for the sections before and after the crack position, and $V_{1}(x, t)$ and $V_{2}(x, t)$ are the amplitudes of bending vibration for the same sections as shown in Figure 2.
The normal functions for the cracked shaft in nondimensional form for both the longitudinal and bending vibration in steady state can be defined as

$$
\begin{array}{r}
\bar{S}_{1}(\bar{x})=B_{1} \cos \left(\bar{H}_{s} \bar{x}\right)+B_{2} \sin \left(\bar{H}_{s} \bar{x}\right) \\
\bar{S}_{2}(\bar{x})=B_{3} \cos \left(\bar{H}_{s} \bar{x}\right)+B_{4} \sin \left(\bar{H}_{s} \bar{x}\right) \\
\bar{V}_{1}(\bar{x})=B_{5} \cosh \left(\bar{H}_{v} \bar{x}\right)+B_{6} \sinh \left(\bar{H}_{v} \bar{x}\right) \\
+B_{7} \cos \left(\bar{H}_{v} \bar{x}\right)+B_{8} \sin \left(\bar{H}_{v} \bar{x}\right) \\
\bar{V}_{2}(\bar{x})=B_{9} \cosh \left(\bar{H}_{v} \bar{x}\right)+B_{10} \sinh \left(\bar{H}_{v} \bar{x}\right) \\
+B_{11} \cos \left(\bar{H}_{v} \bar{x}\right)+B_{12} \sin \left(\bar{H}_{v} \bar{x}\right),
\end{array}
$$

where $\bar{x}=x / L, \bar{S}=S / L, \bar{V}=V / L, \alpha=L_{c} / L, \bar{H}_{s}=\omega L / D_{s}$, $D_{s}=(E / \rho)^{1 / 2}, \bar{H}_{V}=\left(\omega L^{2} / D_{v}\right)^{1 / 2}, D_{v}=(E I / \mu)^{1 / 2}$, and $\mu=A \rho, B_{i},(i=1,12)$. Constants are to be determined from boundary conditions.

Boundary conditions are as follows.

At the left-fixed end

$$
\bar{S}_{1}(0)=0 ; \quad \bar{V}_{1}(0)=0 ; \quad \frac{\partial \bar{V}_{1}}{\partial \bar{X}}(0)=0 .
$$

At the right-fixed end

$$
\bar{S}_{1}(1)=0 ; \quad \bar{V}_{1}(1)=0 ; \quad \frac{\partial \bar{V}_{1}}{\partial \bar{X}}(1)=0 .
$$

At the cracked section

$$
\begin{array}{cl}
\bar{S}_{1}(\alpha)=\bar{S}_{2}(\alpha) ; & \bar{V}_{1}(\alpha)=\bar{V}_{2}(\alpha) ; \\
\frac{\partial^{2} \bar{V}_{1}}{\partial X^{2}}(\alpha)=\frac{\partial^{2} \bar{V}_{2}}{\partial X^{2}}(\alpha) ; \quad & \frac{\partial^{3} \bar{V}_{1}}{\partial X^{3}}(\alpha)=\frac{\partial^{3} \bar{V}_{2}}{\partial X^{3}}(\alpha) .
\end{array}
$$


Also at the cracked section, by force balancing

$$
\begin{aligned}
A E \frac{d S_{1}\left(L_{c}\right)}{d x}=K_{11} & \left(S_{2}\left(L_{c}\right)-S_{1}\left(L_{c}\right)\right) \\
+ & K_{12}\left(\frac{d V_{2}\left(L_{c}\right)}{d x}-\frac{d V_{1}\left(L_{c}\right)}{d x}\right) \\
\Longrightarrow N_{1} N_{2} \bar{S}_{1}^{\prime}(\alpha)= & N_{2}\left(\bar{S}_{2}(\alpha)-\bar{S}_{1}(\alpha)\right) \\
& +N_{1}\left(\bar{V}_{2}^{\prime}(\alpha)-\bar{V}_{1}^{\prime}(\alpha)\right) .
\end{aligned}
$$

Similarly, by moment balancing

$$
\begin{aligned}
E I \frac{d^{2} V_{1}\left(L_{c}\right)}{d x^{2}}=K_{21} & \left(S_{2}\left(L_{c}\right)-S_{1}\left(L_{c}\right)\right) \\
& +K_{22}\left(\frac{d V_{2}\left(L_{c}\right)}{d x}-\frac{d V_{1}\left(L_{c}\right)}{d x}\right) \\
\Longrightarrow N_{3} N_{4} \bar{V}_{1}^{\prime \prime}(\alpha) & =N_{3}\left(\overline{S_{2}}(\alpha)-\overline{S_{1}}(\alpha)\right) \\
& +N_{4}\left(\bar{V}_{2}^{\prime}(\alpha)-\bar{V}_{1}^{\prime}(\alpha)\right),
\end{aligned}
$$

where $N_{1}=A E / L K_{11}, N_{2}=A E / K_{12}, N_{3}=E I / L K_{22}$, and $N_{4}=E I / L^{2} K_{21}$.

The normal functions, (2a), (2b), (2c), and (2d), along with the boundary conditions as mentioned above, yield the characteristic equation of the system as $|\Omega|=0$.

$\Omega$ is a $12 \times 12$ matrix (the Appendix) whose determinant is a function of natural circular frequency $(\omega)$, the nondimensional location of the crack $(\alpha)$, and the local stiffness matrix $(K)$ which in turn is a function of the nondimensional crack depth $\delta_{1}=\left(b_{1} / T\right)$.

\section{Analysis of Neural Controller for Crack Detection}

The presence of damage, in general, in a structure undermines the viability of the structure, leads to shorter life time period, and opens the way for complete failure of the system. Hence, development of an automated method to identify cracks accurately in an engineering application is desirable. As it is known that the cracks present in a mechanical element increase the flexibility, decrease the vibration frequencies, and modify the amplitude of vibration, and those changes can be potentially used to locate the crack positions and crack depths. So it is of interest to design and develop an AI-based technique for online multiple crack diagnosis to avoid catastrophic failure of structural system. In this Section, an intelligent controller has been designed using artificial neural network to detect the presence of a crack in structural members. The proposed neural controller has been modeled with feed forward network trained with back propagation technique. Finally, the results from the controller have been compared with the experimental results to establish the robustness of the proposed neural method.

The back propagation technique can be used to train the multilayer networks. This technique is an approximate steepest algorithm in which the performance of the network is based on mean square error. In order to train the neural network, the weights for each input to the neural system should be so adjusted that the error between the actual output and desired output is minimum. The multilayer neural system would calculate the change in error due to increase or decrease in the weights. The algorithm first computes each error weight by computing the rate of the error changes with the change in synaptic weights. The error in each hidden layer, just before the output layer in a direction opposite to the way activities propagate through the network, have to be computed and fed to the network by back propagation algorithm to minimize the error in the actual output and desired output by adjusting the parameters of the network.

The main features of the neural model are as follows

(1) The inputs to the neuron are assigned with synaptic weights, which in turn affect the decision making ability of the neural network. The inputs to the neuron are called weighted inputs.

(2) These weighted inputs are then summed together in an adder and if they exceed a preset threshold value, the neuron fires. In any other case the neuron does not fire.

(3) An activation function for limiting the amplitude of the output of a neuron has been adopted in the current model. Generally, the normalized amplitude range of the output of a neuron is given as the closed unit interval $[0,1]$ or alternatively $[-1,1]$.

A back propagation neural network controller has been developed for detection of the relative crack location and relative crack depth (Figure 3). The neural network has got six input parameters and two output parameters.

The inputs to the neural network controller are as follows:

$\bar{\Delta}_{n f_{1}}=$ relative first natural frequency,

$\bar{\Delta}_{n f_{2}}=$ relative second natural frequency,

$\bar{\Delta}_{n f_{3}}=$ relative third natural frequency,

$\bar{\Delta}_{m_{1}}=$ relative first mode shape difference;

$\bar{\Delta}_{m_{2}}=$ relative second shape difference,

$\bar{\Delta}_{m_{3}}=$ relative third mode shape difference.

The outputs from the neural network are as follows;

relative crack location $=\alpha$ and relative crack depth $=$ $\delta_{1}$.

The back propagation neural network controller has got 5 layers (i.e., input layer, output layer, and three hidden layers). The neurons associated with the input and output layers are six and two, respectively. The input layer neurons represent relative deviation of the first three natural frequencies and the first three relative mode shape differences. The output layer neurons represent relative crack location and relative crack depth. The neurons are taken in order to give the neural network a diamond shape. 
TABLE 1: Examples of the training patterns for training of the neural network controller.

\begin{tabular}{|c|c|c|c|c|c|c|c|c|}
\hline \multirow[t]{2}{*}{ Serial number } & \multicolumn{6}{|c|}{ Input to the neural network controller } & \multicolumn{2}{|c|}{$\begin{array}{l}\text { Desired output from the neural } \\
\text { network controller }\end{array}$} \\
\hline & $\bar{\Delta}_{n f_{1}}$ & $\bar{\Delta}_{n f_{2}}$ & $\bar{\Delta}_{n f_{3}}$ & $\bar{\Delta}_{m_{1}}$ & $\bar{\Delta}_{m_{2}}$ & $\bar{\Delta}_{m_{3}}$ & $\alpha$ & $\delta_{1}$ \\
\hline 1 & 0.9184 & 0.9232 & 0.9602 & 0.2007 & 0.4218 & 0.2035 & 0.149 & 0.505 \\
\hline 2 & 0.9264 & 0.9772 & 0.9854 & 0.1426 & 0.2233 & 0.1821 & 0.099 & 0.412 \\
\hline 3 & 0.9430 & 0.9806 & 0.9862 & 0.1008 & 0.1624 & 0.2064 & 0.172 & 0.398 \\
\hline 4 & 0.9456 & 0.9810 & 0.9876 & 0.0958 & 0.1593 & 0.2052 & 0.072 & 0.199 \\
\hline 5 & 0.9578 & 0.9862 & 0.9878 & 0.0709 & 0.1492 & 0.1968 & 0.273 & 0.536 \\
\hline 6 & 0.9662 & 0.9872 & 0.9894 & 0.0460 & 0.1305 & 0.1936 & 0.214 & 0.249 \\
\hline 7 & 0.9678 & 0.9908 & 0.9936 & 0.0351 & 0.1219 & 0.1604 & 0.382 & 0.513 \\
\hline 8 & 0.9726 & 0.9922 & 0.9938 & 0.0182 & 0.0458 & 0.1319 & 0.425 & 0.491 \\
\hline 9 & 0.9804 & 0.9936 & 0.9964 & 0.0147 & 0.0299 & 0.0912 & 0.503 & 0.540 \\
\hline 10 & 0.9882 & 0.9980 & 0.9982 & 0.0063 & 0.0132 & 0.0349 & 0.489 & 0.430 \\
\hline
\end{tabular}

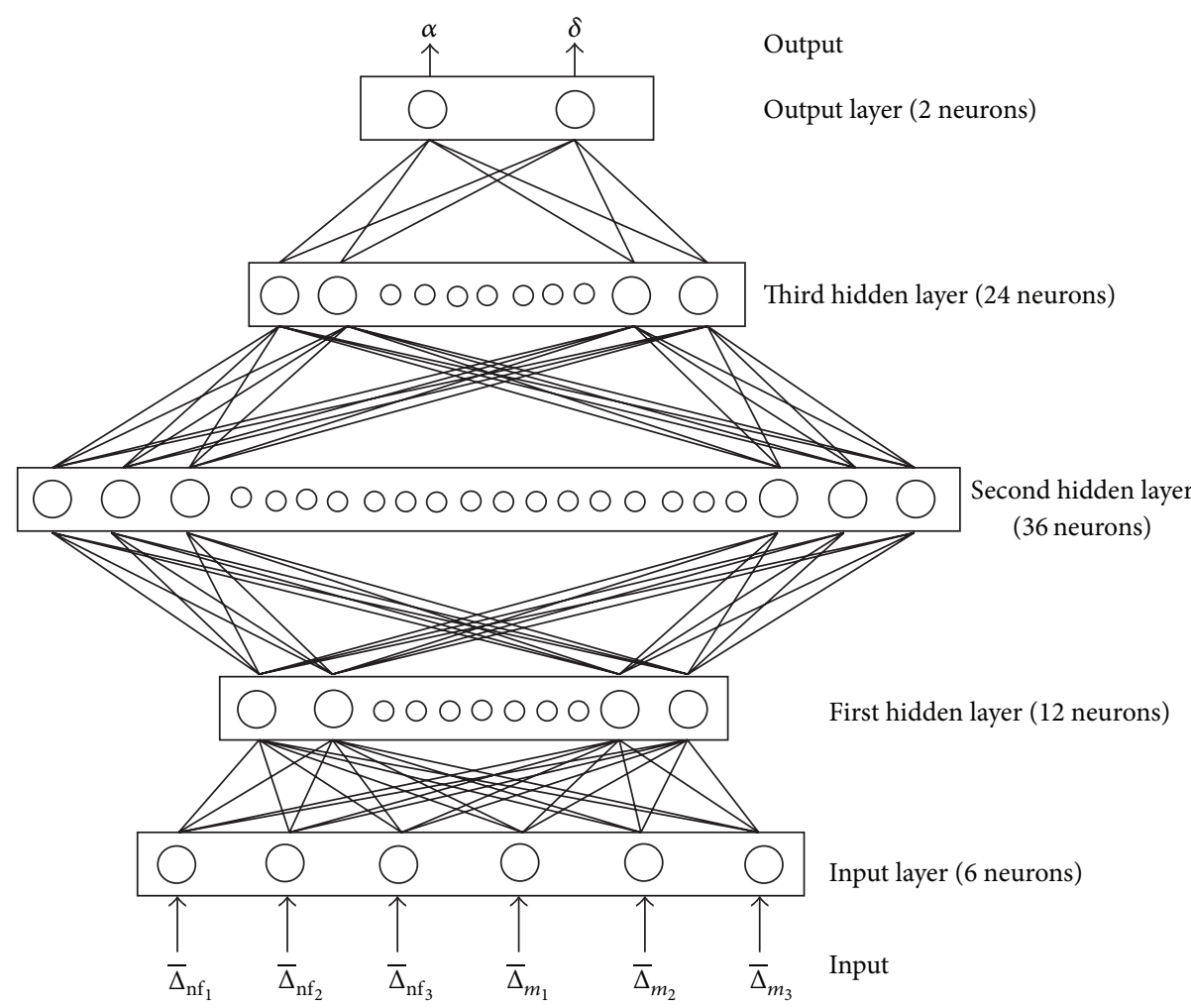

FIGURE 3: Five-layer neural network controller for crack detection.

TABLE 2: Material properties of aluminium-alloy, 2014- $\mathrm{T}_{4}$.

\begin{tabular}{lcccc}
\hline $\begin{array}{l}\text { Young's } \\
\text { modulus, } E\end{array}$ & Density, $\rho$ & $\begin{array}{c}\text { Poisson's } \\
\text { ratio, } \mu\end{array}$ & $\begin{array}{c}\text { Length, } \\
L\end{array}$ & $\begin{array}{c}\text { Diameter, } \\
D\end{array}$ \\
\hline $72.4 \mathrm{GPa}$ & $2.8 \mathrm{gm} / \mathrm{cc}$ & 0.33 & $800 \mathrm{~mm}$ & $150 \mathrm{~mm}$ \\
\hline
\end{tabular}

The relative crack depth $\left(\delta_{1}=b_{1} / D\right)=$ varies from 0.05 to 0.8 .

The relative crack location $\left(\alpha=L_{c} / L\right)=$ varies from 0.0125 to 0.95 .

3.1. Neural Controller Mechanism for Crack Detection. The neural network used is a five-layer perceptron [11]. The chosen number of layers was found empirically to facilitate training. The input layer has six neurons, three for the first three relative natural frequencies and the other three for the first three relative mode shape differences. The output layer has two neurons, which represent relative crack location and relative crack depth. These numbers of hidden neurons are also found empirically. Figure 4 depicts the neural network with its input and output signals.

The neural network is trained with 500 patterns representing typical scenarios, some of which are depicted in Table 1. The neural network is trained to give outputs such 
TABLE 3: Comparison of results between neural network controller, numerical analysis, and experimental setup.

\begin{tabular}{lccccccccccccc}
\hline \multirow{2}{*}{ Slerial number } & $\bar{\Delta}_{n f_{1}}$ & $\bar{\Delta}_{n f_{2}}$ & $\bar{\Delta}_{n f_{3}}$ & $\bar{\Delta}_{m_{1}}$ & $\bar{\Delta}_{m_{2}}$ & $\bar{\Delta}_{m_{3}}$ & \multicolumn{2}{c}{ Neural network controller } & \multicolumn{2}{c}{ Numerical } & \multicolumn{2}{c}{ Experimental } \\
& 0.9429 & 0.9534 & 0.9554 & 0.1354 & 0.1186 & 0.1579 & 0.195 & 0.071 & 0.194 & 0.067 & 0.197 & 0.072 \\
2 & 0.9265 & 0.9457 & 0.9523 & 0.1984 & 0.1623 & 0.1961 & 0.414 & 0.079 & 0.411 & 0.077 & 0.413 & 0.082 \\
3 & 0.9217 & 0.9525 & 0.9558 & 0.0907 & 0.0139 & 0.0387 & 0.526 & 0.156 & 0.516 & 0.156 & 0.546 & 0.154 \\
4 & 0.9345 & 0.9555 & 0.9552 & 0.0691 & -0.0412 & 0.0949 & 0.374 & 0.183 & 0.378 & 0.183 & 0.375 & 0.184 \\
5 & 0.9433 & 0.9563 & 0.9583 & 0.0051 & -0.4339 & 0.1286 & 0.218 & 0.233 & 0.222 & 0.231 & 0.221 & 0.235 \\
6 & 0.9313 & 0.9541 & 0.9404 & 0.0973 & 0.0336 & 0.2052 & 0.531 & 0.281 & 0.534 & 0.277 & 0.524 & 0.28 \\
7 & 0.9409 & 0.9456 & 0.9497 & 0.0363 & 0.1283 & 0.1997 & 0.432 & 0.397 & 0.433 & 0.396 & 0.429 & 0.397 \\
8 & 0.9432 & 0.9396 & 0.9512 & 0.0449 & 0.1577 & 0.1961 & 0.475 & 0.415 & 0.477 & 0.415 & 0.475 & 0.415 \\
9 & 0.9444 & 0.9192 & 0.9563 & -0.016 & 0.1611 & 0.1982 & 0.408 & 0.493 & 0.409 & 0.491 & 0.408 & 0.494 \\
10 & 0.9419 & 0.9277 & 0.9554 & 0.0189 & 0.2279 & 0.1753 & 0.516 & 0.523 & 0.521 & 0.524 & 0.514 & 0.522 \\
\hline
\end{tabular}

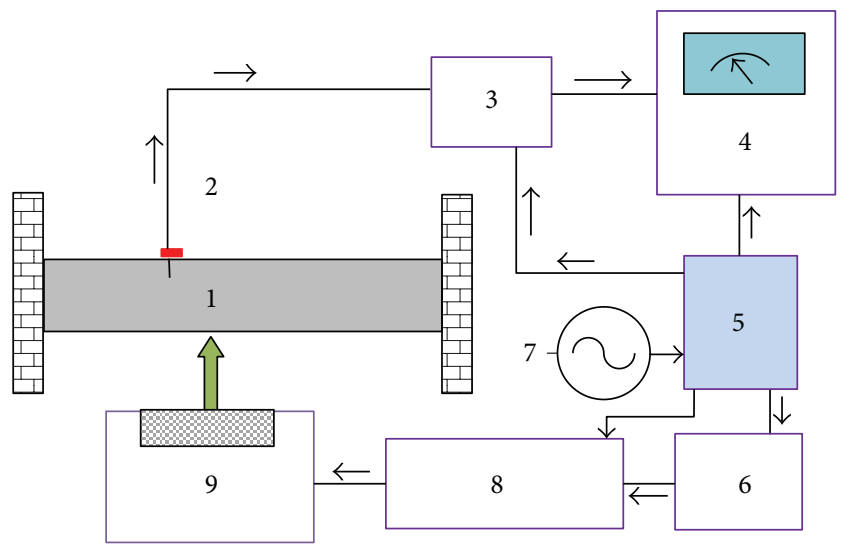

(1) Fixed-fixed beam specimen

(2) Vibration pickup (accelerometer)

(3) Vibration analyzer (pulse lite type)

(4) Vibration indicator with software (PULS LabShop software)

(5) Distribution box

FIGURE 4: Schematic block diagram of experimental setup for fixedfixed cracked beam.

as relative crack depth and relative crack location. During training and during normal operation, the input patterns fed to the neural network comprise the following components.

$\Delta_{1}^{\{1\}}, \Delta_{2}^{\{1\}}$, and $\Delta_{3}^{\{1\}}$ are the relative deviations of first, second, and third natural frequencies and $\Delta_{4}^{\{1\}}, \Delta_{5}^{\{1\}}$, and $\Delta_{6}^{\{1\}}$ are the relative deviations of first, second, and third mode shape differences.

These input values are distributed to the hidden neurons which generate outputs given by [11]

$$
\Delta_{j}^{\{\text {lay }\}}=g\left(V_{j}^{\{\text {lay }\}}\right),
$$

where

$$
V_{j}^{\{\text {lay }\}}=\sum_{i} w_{j i}^{\{\text {lay }\}} \cdot \Delta_{i}^{\{\text {lay }-1\}}
$$

lay = layer number (2 or 4$)$,

$j=$ label for $j$ th neuron in hidden layer "lay" and $i=$ label for $i$ th neuron in hidden layer "lay-1",

$w_{j i}^{\{l \text { lay }}=$ weight of the connection from neuron $i$ in layer "lay-1" to neuron $j$ in layer "lay".

$g(\cdot)=$ activation function, chosen in this work as the hyperbolic tangent function as

$$
g(x)=\frac{e^{x}-e^{-x}}{e^{x}+e^{-x}}
$$

During training, the network output $\theta_{\text {actual, } n}(i=1,2)$ may differ from the desired output $\theta_{\text {desired, } n}(n=1,2)$ as specified in the training pattern presented to the network. A measure of the performance of the network is the instantaneous sumsquared difference between $\theta_{\text {desired }, n}$ and $\theta_{\text {actual }, n}$ for the set of presented the following training patterns:

$$
\text { error }=\frac{1}{2} \sum_{\text {all training patterns }}\left(\theta_{\text {desired }, n}-\theta_{\text {actual }, n}\right)^{2}
$$

where $\theta_{\text {actual }, n}(n=1)$ represents relative crack location $(\alpha)$, and $\theta_{\text {actual }, n}(n=2)$ represents relative crack depth $\left(\delta_{1}\right)$.

The error back propagation method is employed to train the network. This method requires the computation of local error gradients in order to determine appropriate weight corrections to reduce error. For the output layer, the error gradient $\exists^{\{5\}}$ is

$$
\exists^{\{5\}}=g^{\prime}\left(V_{1}^{\{5\}}\right)\left(\theta_{\text {desired }, n}-\theta_{\text {actual }, n}\right) .
$$

The local gradient for neurons in hidden layer lay is given by

$$
\exists_{j}^{\{\text {lay }\}}=g^{\prime}\left(V_{j}^{\{\text {lay }\}}\right)\left(\sum_{k} \exists_{k}^{\{\mathrm{lay}+1\}} W_{k j}^{\{\mathrm{lay}+1\}}\right) .
$$




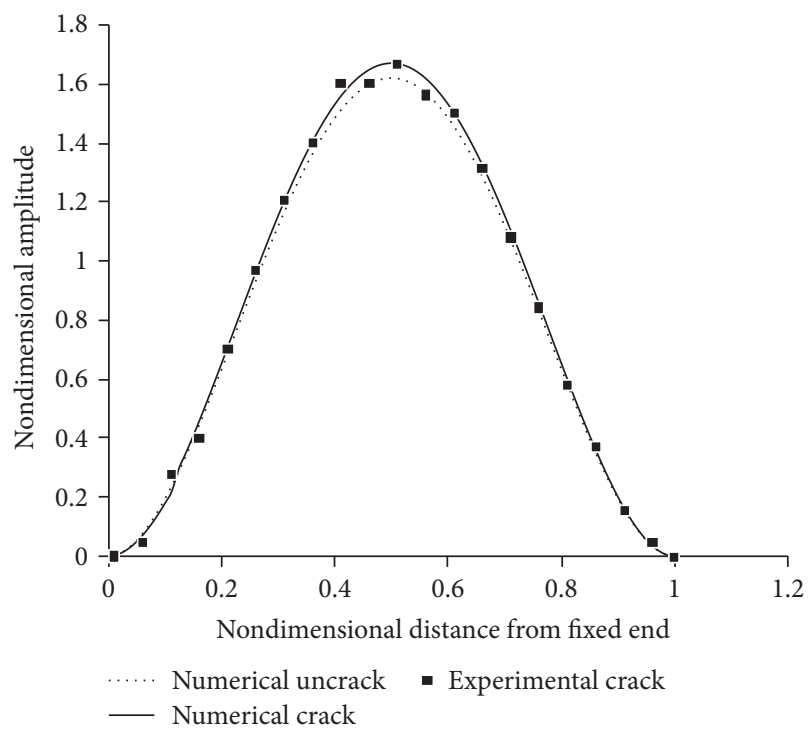

(a)

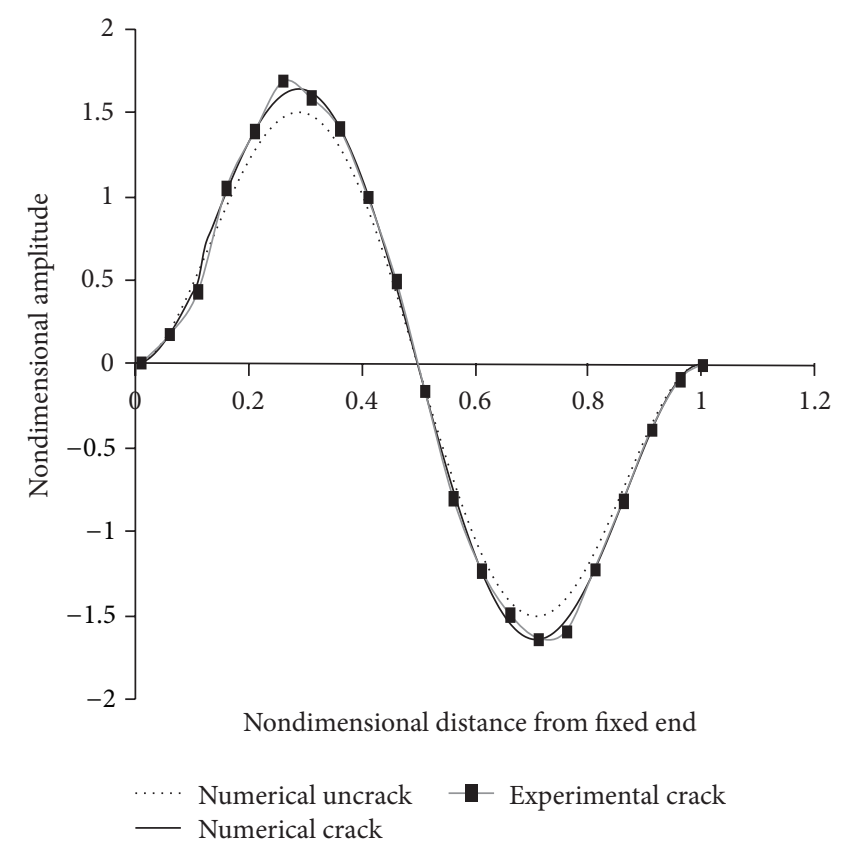

(c)

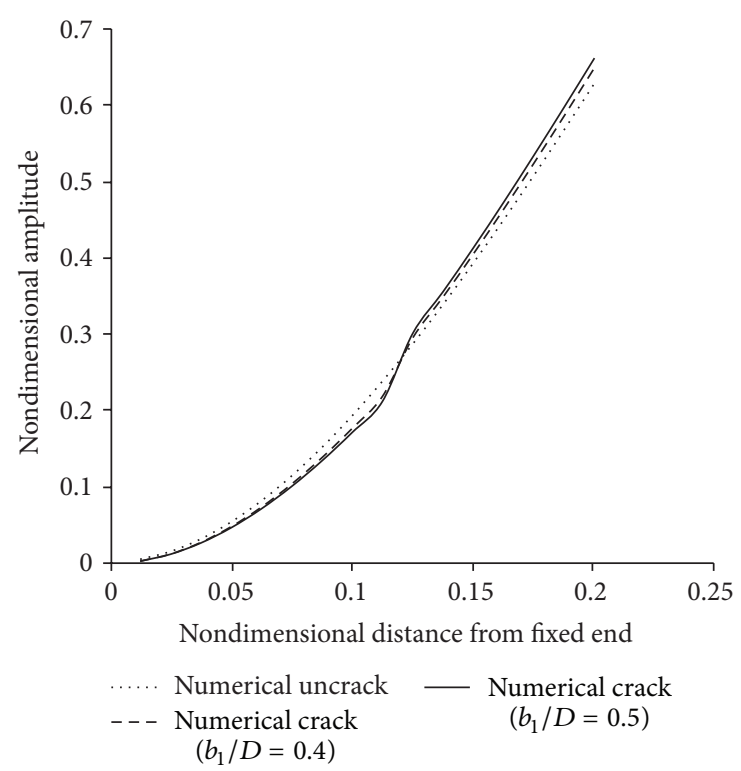

(b)

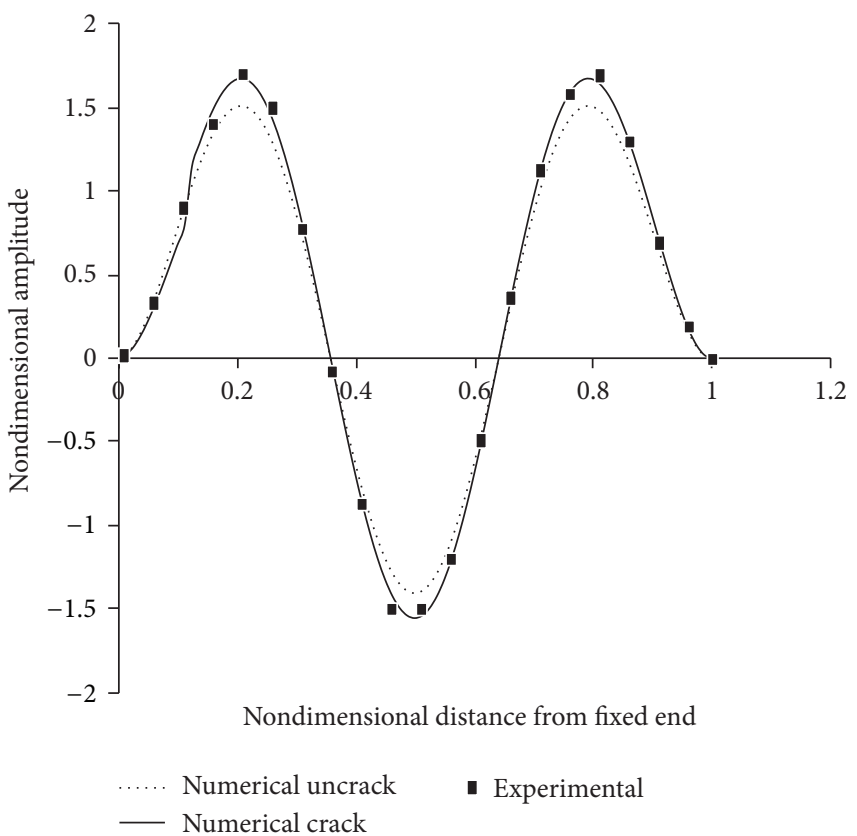

(d)

Figure 5: (a) Nondimensional amplitude versus nondimensional distance from left end (1st mode of vibration), $b_{1} / D=0.4, L_{c} / L=0.125$. (b) Magnified view of Figure 5(a) at the vicinity of the crack location. (c) Nondimensional amplitude versus nondimensional distance from left end (2nd mode of vibration), $b_{1} / D=0.4, L_{c} / L=0.125$. (d) Nondimensional amplitude versus nondimensional distance from left end (3rd mode of vibration), $b_{1} / D=0.4, L_{c} / L=0.125$.

The synaptic weights are updated according to the following expressions:

$$
\begin{gathered}
w_{j i}(t+1)=w_{j i}(t)+(\Delta w)_{j i}(t+1), \\
(\Delta w)_{j i}(t+1)=\beta(\Delta w)_{j i}(t)+\eta \exists_{j}^{\{\text {lay }\}} \Delta_{i}^{\{\text {lay- } 1\}},
\end{gathered}
$$

$\beta=$ momentum coefficient (chosen empirically as 0.2 in this work), $\eta=$ learning rate (chosen empirically as 0.35 in this work),

$t=$ iteration number, each iteration consisting of the presentation of a training pattern and correction of the weights.

The final output from the neural network is

$$
\theta_{\text {actual }, n}=g\left(V_{n}^{\{5\}}\right), \quad \text { where } V_{n}^{\{5\}}=\sum_{i} w_{n i}^{\{5\}} \Delta_{i}^{\{4\}} \text {. }
$$




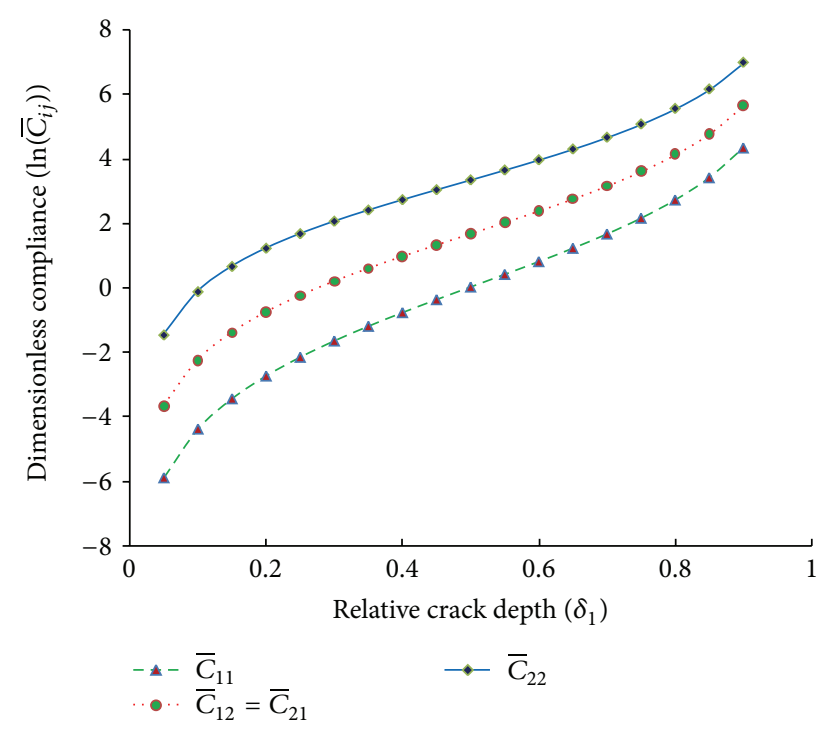

FIgURE 6: Dimensionless compliance $\left(\ln \left(\bar{C}_{i j}\right)\right)$ versus relative crack depth $\left(\delta_{1}\right)$.

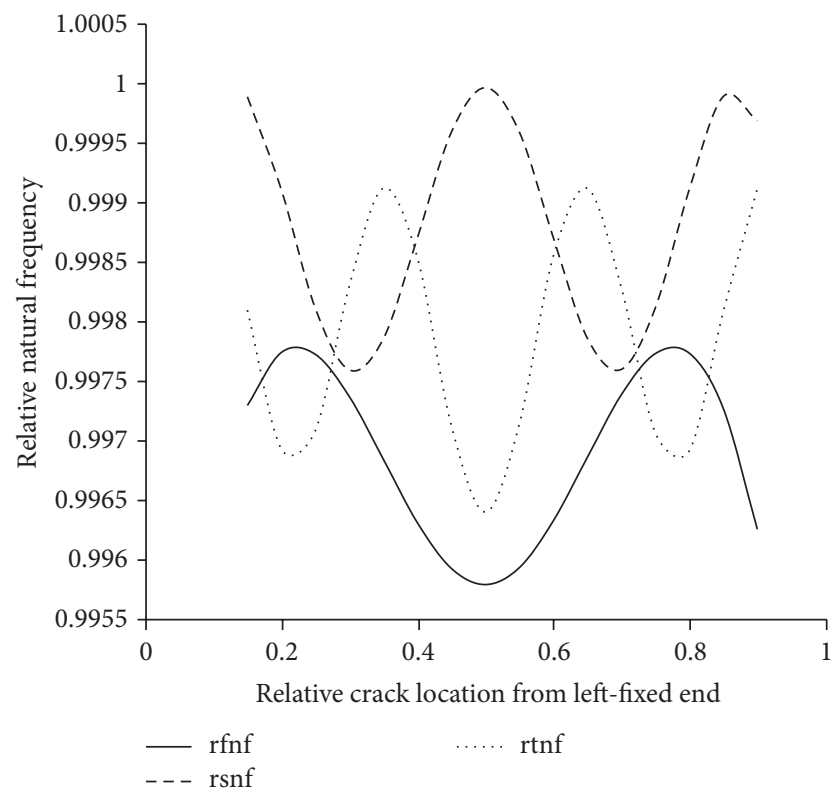

FIGURE 7: Relative natural frequency versus relative crack location from the fixed end $b_{1} / T=0.3$.

\section{Experimental Validation}

Experiments are performed to determine the natural frequencies and mode shapes for different crack depths on aluminum shaft specimen having $800 \mathrm{~mm}$ in length and $150 \mathrm{~mm}$ in diameter on the experimental setup shown in schematic diagram Figure 4. The amplitude of transverse vibration at different locations along the length of the aluminum beam is recorded by positioning the vibration pickup and tuning the vibration generator at the corresponding resonant frequencies. These results for first three modes are plotted in Figure 5. Corresponding numerical results for the

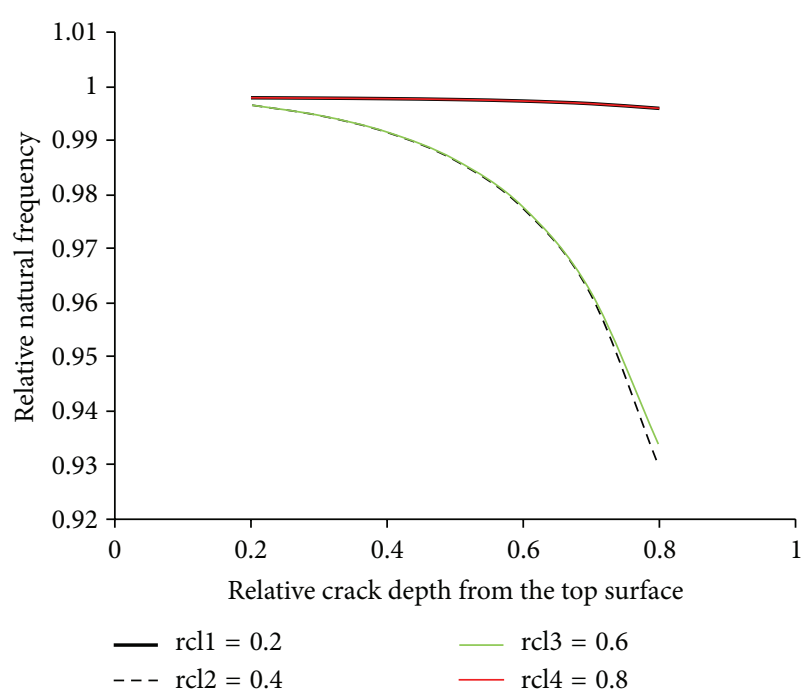

FIGURE 8: Relative natural frequency versus relative crack depth from the top surface (1st mode vibration).

cracked and uncracked beam are also presented in the same graph for comparison.

4.1. Shaft Specimen Specification. In the current investigation, using both numerical and experimental analysis of a cracked shaft, the following (Table 2) specifications are being considered.

\section{Discussion}

In this section, the results from the theoretical neural network and experimental analysis performed on the cracked fixedfixed shaft are discussed. Figure 1 presents the cracked shaft with its cross-sectional view. A comparison between the first three mode shapes of numerical crack, numerical un-crack, and the magnified view of the shaft (at the vicinity of the crack location) are also shown in Figure 5. A neural controller has been shown in Figure 3. Examples of the training patterns for training of the neural network controller are presented in Table 1. An experimental setup has been developed (Figure 4) to perform experiments on aluminum shaft specimen for validation of results obtained from theoretical and neural network analysis. The results from theoretical analysis and experimental setup are compared and the results for cracked and uncracked shaft are presented in Figure 5.

The dimensionless compliances $\left(\bar{C}_{11}, \bar{C}_{12}=\bar{C}_{21}, \bar{C}_{22}\right)$ increase with the increase in relative crack depth as shown in Figure 6. Figure 7 explains how the natural frequencies vary with the location of crack. Since fixed-fixed beam is geometrically symmetric, the variations are symmetric between left half and right half. Higher modes of vibration show both maximaly and minimaly more numbers of times because of more number of nodes. As shown in Figure 8, natural frequencies are in decreasing trend with depth of crack. The variations of frequencies are sharper for cracks located far away from either end of the beam. 
The results of the numerical analysis, the neural network method, and the experimental method are shown in Table 3. This proposed NN scheme shows very high accuracy in predicting damage location and severity. The high detection performance, combined with the simple computation structure and high learning efficiency, could lead to a promising real-time damage detection system.

\section{Conclusions}

The conclusions drawn from the above analyses are described in this section. From the analysis of the vibration characteristics a clear-cut deviation of the mode shapes and natural frequencies has been observed for the cracked and uncracked shaft. Comparisons of results from neural network method and theoretical and experimental analysis have been carried out and they show a very good agreement. This method can be utilized for condition monitoring of cracked vibrating structures, and, in future, hybrid techniques can be developed for fault identification in damaged vibrating structures.

\section{Appendix}

$$
Q=
$$$$
\left[\begin{array}{cccccccccccc}
1 & 0 & 1 & 0 & 0 & 0 & 0 & 0 & 0 & 0 & 0 & 0 \\
0 & 1 & 0 & 1 & 0 & 0 & 0 & 0 & 0 & 0 & 0 & 0 \\
0 & 0 & 0 & 0 & G_{3} & G_{4} & -G_{7} & -G_{8} & 0 & 0 & 0 & 0 \\
0 & 0 & 0 & 0 & G_{4} & G_{3} & G_{8} & -G_{7} & 0 & 0 & 0 & 0 \\
G_{1} & G_{2} & -G_{5} & -G_{6} & -G_{1} & -G_{2} & G_{5} & G_{6} & 0 & 0 & 0 & 0 \\
G_{2} & G_{1} & G_{6} & -G_{5} & -G_{2} & -G_{1} & -G_{6} & G_{5} & 0 & 0 & 0 & 0 \\
G_{1} & G_{2} & G_{5} & G_{6} & -G_{1} & -G_{2} & -G_{5} & -G_{6} & 0 & 0 & 0 & 0 \\
S_{1} & S_{2} & S_{3} & S_{4} & -G_{2} & -G_{1} & G_{6} & -G_{5} & S_{5} & S_{6} & S_{7} & S_{8} \\
0 & 0 & 0 & 0 & 0 & 0 & 0 & 0 & 1 & 0 & 0 & 0 \\
0 & 0 & 0 & 0 & 0 & 0 & 0 & 0 & 0 & 0 & -T_{8} & T_{7} \\
0 & 0 & 0 & 0 & 0 & 0 & 0 & 0 & -T_{6} & T_{5} & T_{6} & -T_{5} \\
S_{9} & S_{10} & S_{11} & S_{12} & S_{13} & S_{14} & S_{15} & S_{16} & S_{17} & S_{18} & -T_{5} & -T_{6}
\end{array}\right],
$$

where

$$
\begin{aligned}
& S_{1}=G_{2}+N_{3} \bar{H}_{V} G_{1}, \quad S_{2}=G_{1}+N_{3} \bar{H}_{V} G_{2}, \\
& S_{3}=-G_{6}-N_{3} \bar{H}_{V} G_{5}, \quad S_{4}=G_{5}-N_{3} \bar{H}_{V} G_{6}, \\
& S_{5}=\frac{N_{34}}{\bar{H}_{V}} T_{5}, \quad S_{6}=\frac{N_{34}}{\bar{H}_{V}} T_{6}, \quad S_{7}=\frac{-N_{34}}{\bar{H}_{V}} T_{5}, \\
& S_{8}=\frac{-N_{34}}{\bar{H}_{V}} T_{6}, \quad S_{9}=N_{12} \bar{H}_{V} G_{2}, \quad S_{10}=N_{12} \bar{H}_{V} G_{1}, \\
& S_{11}=-N_{12} \bar{H}_{V} G_{6}, \quad S_{12}=N_{12} \bar{H}_{V} G_{5}, \\
& S_{13}=-N_{12} \bar{H}_{V} G_{2}, \quad S_{14}=-N_{12} \bar{H}_{V} G_{1}, \\
& S_{15}=N_{12} \bar{H}_{V} G_{6}, \quad S_{16}=-N_{12} \bar{H}_{V} G_{5}, \\
& S_{17}=T_{5}-N_{1} \bar{H}_{S} T_{6}, \quad S_{18}=T_{6}+6 N_{1} \bar{H}_{S} T_{5}, \\
& G_{1}=\operatorname{Cosh}\left(\bar{H}_{V} \alpha\right), \quad G_{2}=\operatorname{Sinh}\left(\bar{H}_{V} \alpha\right), \\
& G_{3}=\operatorname{Cosh}\left(\bar{H}_{V}\right), \quad G_{4}=\operatorname{Sinh}\left(\bar{H}_{V}\right), \\
& G_{5}=\operatorname{Cos}\left(\bar{H}_{V} \alpha\right), \quad G_{6}=\operatorname{Sin}\left(\bar{H}_{V} \alpha\right),
\end{aligned}
$$

$$
\begin{aligned}
G_{7} & =\operatorname{Cosh}\left(\bar{H}_{V}\right), \quad G_{8}=\operatorname{Sin}\left(\bar{H}_{V}\right), \\
T_{5} & =\operatorname{Sin}\left(\bar{H}_{S} \alpha\right), \quad T_{6}=\operatorname{Sin}\left(\bar{H}_{S} \alpha\right), \\
T_{7} & =\operatorname{Cos}\left(\bar{H}_{S}\right), \quad T_{8}=\operatorname{Sin}\left(\bar{H}_{S}\right), \\
N_{12} & =\frac{N_{1}}{N_{2}}, \quad N_{34}=\frac{N_{3}}{N_{4}} .
\end{aligned}
$$

\section{References}

[1] R. D. Adams, D. Walton, J. E. Flitcroft, and D. Short, "Composite Reliability, Philadelphia: American Society for Testing Materials," Vibration testing as a nondestructive test tool for composite materials, ASTM STP 580, pp.159-175, 1975.

[2] H. Tada, P. C. Paris, and G. R. Irwin, The Stress Analysis of Cracks Hand Book, Del Research, Hellertown, Pennsylvania, 1973.

[3] A. S. Sekhar and B. S. Prabhu, "Crack detection and vibration characteristics of cracked shafts," Journal of Sound and Vibration, vol. 157, no. 2, pp. 375-381, 1992.

[4] G. Paviglianiti, F. Pierri, F. Caccavale, and M. Mattei, "Robust fault detection and isolation for proprioceptive sensors of robot manipulators," Mechatronics, vol. 20, no. 1, pp. 162-170, 2010.

[5] R. K. Behera, D. R. K. Parhi, and S. K. Sahu, "Vibration analysis of a cracked rotor surrounded by viscous liquid," JVC/Journal of Vibration and Control, vol. 12, no. 5, pp. 465-494, 2006.

[6] K. Wang, D. J. Inman, and C. R. Farrar, "Modeling and analysis of a cracked composite cantilever beam vibrating in coupled bending and torsion," Journal of Sound and Vibration, vol. 284, no. 1-2, pp. 23-49, 2005.

[7] J. H. Lee, "Identification of multiple cracks in a beam using natural frequencies," Journal of Sound and Vibration, vol. 320, no. 3, pp. 482-490, 2009.

[8] M. Sahin and R. A. Shenoi, "Quantification and localisation of damage in beam-like structures by using artificial neural networks with experimental validation," Engineering Structures, vol. 25, no. 14, pp. 1785-1802, 2003.

[9] B. Sahoo and D. Maity, "Damage assessment of structures using hybrid neuro-genetic algorithm," Applied Soft Computing Journal, vol. 7, no. 1, pp. 89-104, 2007.

[10] S. Suresh, S. N. Omkar, R. Ganguli, and V. Mani, "Identification of crack location and depth in a cantilever beam using a modular neural network approach," Smart Materials and Structures, vol. 13, no. 4, pp. 907-915, 2004.

[11] S. Haykin and Neural Networks, A Comprehensive Foundation, Pearson Education, 2006. 

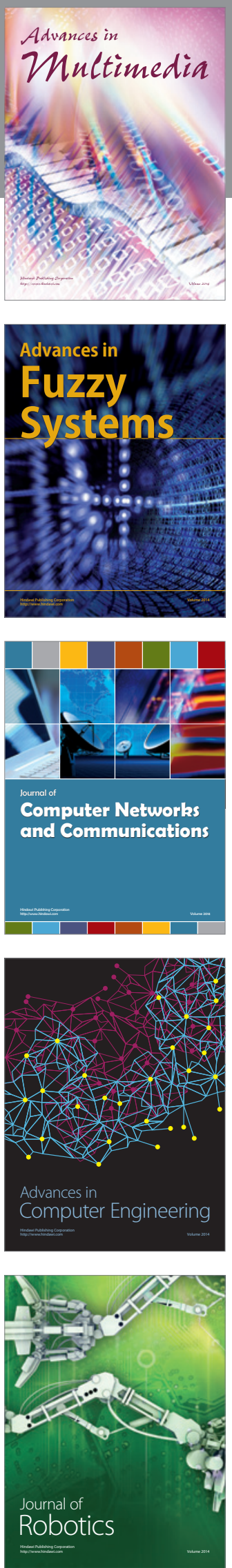

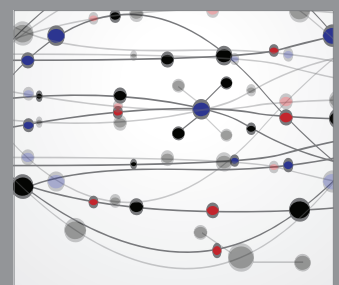

The Scientific World Journal
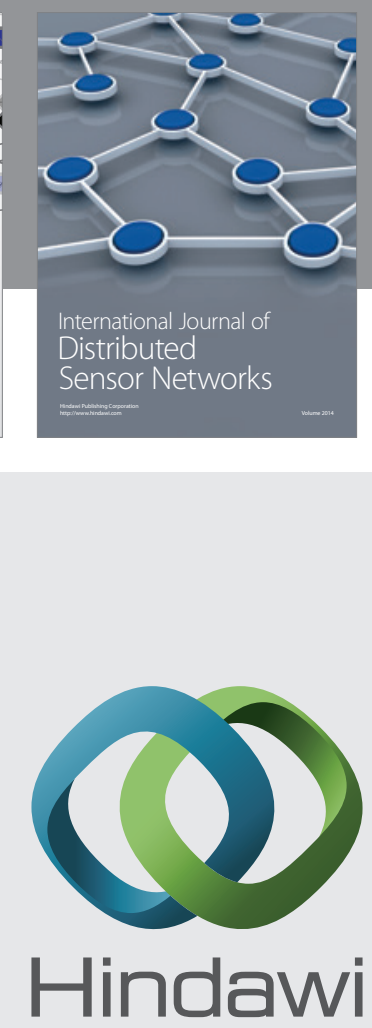

Submit your manuscripts at

http://www.hindawi.com
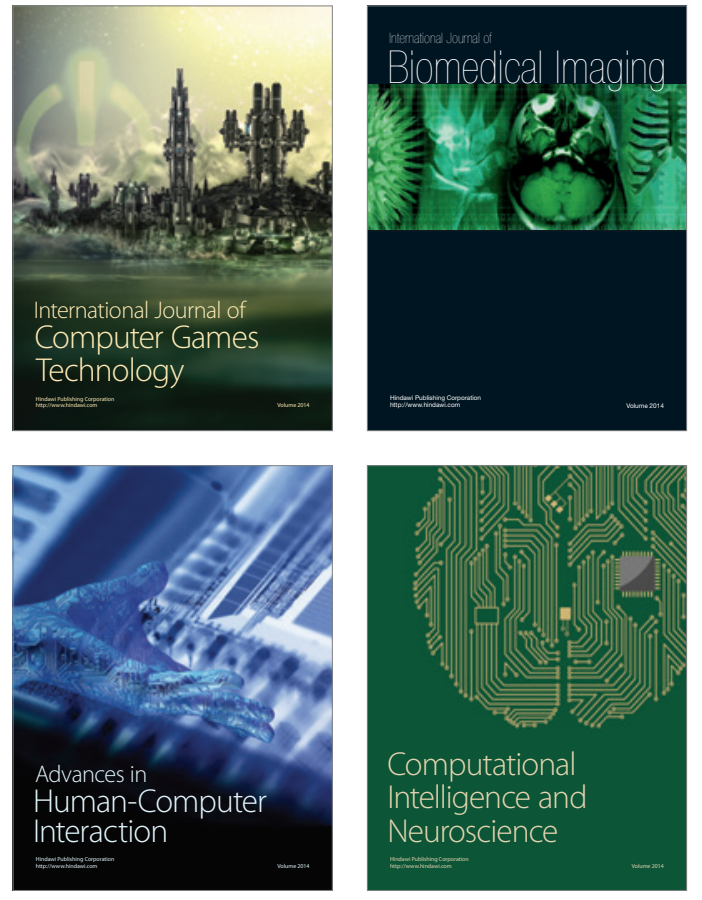
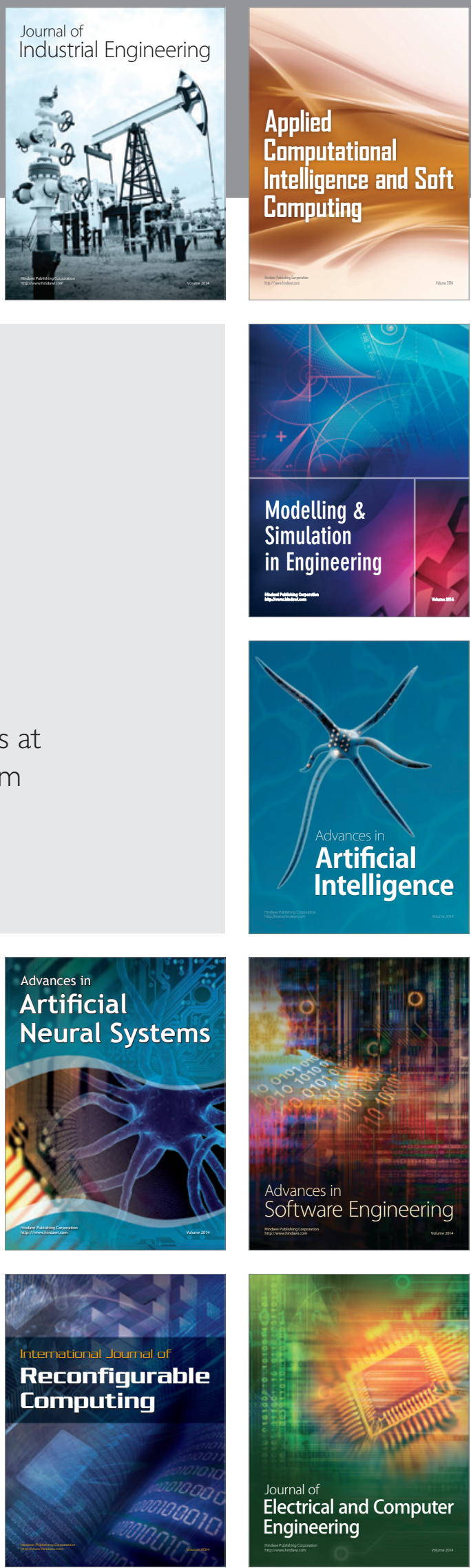